\title{
A Single Leg Switched PWM Method for Three-phase H-Bridge Voltage Source Converters
}

\author{
Osman S. Senturk, Student Member, IEEE \\ Stig Munk-Nielsen, Member, IEEE \\ Remus Teodorescu, Senior Member, IEEE \\ Aalborg University \\ Department of Energy Technology \\ Pontoppidanstraede 101 \\ Aalborg, 9220, Denmark \\ oss@iet.aau.dk,smn@iet.aau.dk,ret@iet.aau.dk
}

\author{
Lars Helle \\ Vestas Wind Systems \\ Pontoppidanstraede 101 \\ Aalborg, 9220, Denmark \\ lah@vestas.com
}

\author{
Pedro Rodriguez \\ Member, IEEE \\ Technical University of Catalonia \\ Dept. of Electrical Engineering \\ C. Colom 1 \\ Terrassa, 08222, Spain \\ prodriguez@ee.upc.edu
}

\begin{abstract}
This paper proposes a single leg switched or a hybrid PWM (HPWM) method for three-phase three-level HBridge Voltage Source Converters (3L-HB-VSCs). By means of the proposed modulation, a $3 \mathrm{~L}-\mathrm{HB}-\mathrm{VSC}$ can generate the same output as a three-level neutral point clamped (3L-NPC) VSC with phase disposition (PD) PWM provided that the outputs of 3L-HBVSC are isolated by transformers or connected to open winding machines. Thus, the proposed method is called PD-HPWM. Moreover, it is emphasized that 3L-HB-VSC with HPWM utilizes its switches similar to 3L-NPC-VSC. Compared to 3L-NPC-VSCs, 3L-HB-VSCs (without neutral point clamping diodes) have simpler, more modular, and more reliable $2 \mathrm{~L}$ circuit structure. Therefore, this method encourages the use of $3 \mathrm{~L}-\mathrm{HB}-\mathrm{VSC}$ in the applications utilizing transformers such as grid-side converters of multi-MW wind turbines. The proposed PWM method's performance is demonstrated by the simulations of a $6 \mathrm{MW}$ wind turbine's grid connection and experimentally verified via a smallscale prototype.
\end{abstract}

Index Terms-- H-Bridge, hybrid modulation, neutral point clamped, multilevel converters, wind energy

\section{INTRODUCTION}

H-bridge (HB) voltage source converters (VSCs) have been mostly utilized in DC/DC converter systems [1] and singlephase AC applications [2]. In three-phase applications, with $\mathrm{AC}$ side isolation transformers as shown in Fig. 1, HB-VSCs have been utilized in Series Active Filter (SAF) [3], Dynamic Voltage Restorer (DVR) [4], and STATCOM [5] applications. Moreover, the three-phase HB-VSC topology or its close variants have been used to drive open winding induction machines [6]-[9] and dual three-phase induction machines [10]. Note that in these induction machine drive systems, these converters are considered as two 2L three-phase VSCs. In addition to these studies, the cascaded connections of $\mathrm{H}$-bridge (CHB) VSCs have been investigated in medium voltage motor drive applications [11], [12].

The modulation of single-phase HB-VSC has been thoroughly studied in the literature [1], [11]. In most applications, three-level (3L) modulation, which is called unipolar pulse width modulation (UPWM), is preferred to the two-level (2L) modulation, which is also called bipolar PWM (BPWM), because UPWM ensures the half of the $d v / d t$ output voltage stress that BPWM results in. As well as UPWM, single phase-leg switched modulation or hybrid PWM (HPWM) generates 3L output voltage [11], [13]. Nevertheless, these PWM methods have not been studied for three-phase HBVSCs elaborately. For the aforementioned 'two 2L VSCs' variants of the three-phase HB-VSC, there are PWM studies which aim at control of dual three-phase induction machines [10], common mode voltage elimination of an open winding induction machine [7], and balancing the power drawn from two isolated DC sources in an open winding induction machine drive system [8]. In [9], it has been demonstrated that by means of a space vector based PWM method a 'cascaded 2L converter' is able to generate the same converter performance as 3L neutral-point-clamped (NPC) VSC (Fig. 2), which has been thoroughly studied in the literature since it was introduced in 1981 [14]. Nonetheless, the correlation between the three-phase HB-VSC (or so-called 3L-HB-VSC hereafter in this paper) with HPWM and 3L-NPC-VSC has not been studied in the literature.

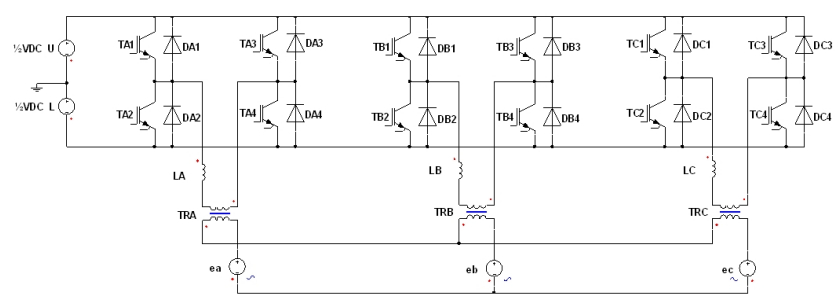

Fig. 1. 3L-HB-VSC with a transformer for grid connection

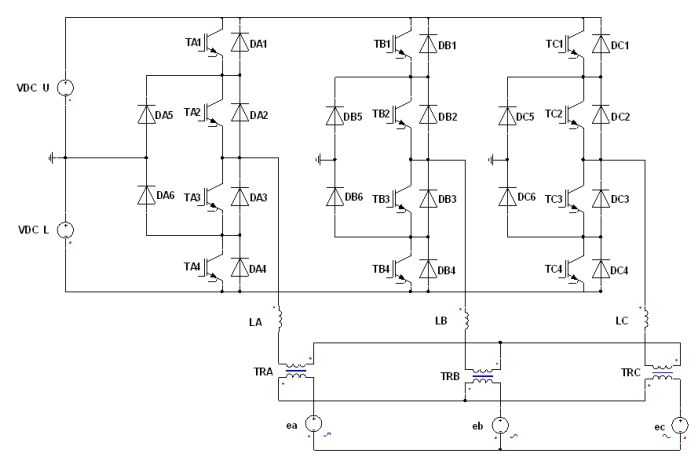

Fig. 2. 3L-NPC-VSC with a transformer for grid connection 
This study demonstrates that a 3L-HB-VSC with HPWM generates the same output voltage as an 3L-NPC-VSC with phase opposition disposition PWM (POD-PWM), and then it proposes a carrier-based HPWM in order 3L-HB-VSC to generate the same output voltage as $3 \mathrm{~L}-\mathrm{NPC}$-VSC with phase disposition PWM (PD-PWM) (or its space vector equivalent of Nearest Triangle Vector (NTV) PWM when zero-sequence triangular voltage is added to three-phase reference voltages). Thus, the former HPWM method will be called POD-HPWM and the proposed method will be called PD-HPWM hereafter in this paper. It should be noted that in a three-phase three-wire system, PD type PWM results in less harmonic distortion than POD type PWM due to the elimination of the common mode harmonic terms in line-to-line voltages [11]; therefore, the existence of the proposed PD-HPWM carries more significance than POD-HPWM particularly for low switching frequency applications where this elimination makes significant difference in harmonic spectrum. Also, this study emphasizes on the capability of 3L-HB-VSC with HPWM to utilize its switches similarly to 3L-NPC-VSC.

In this paper, first, POD-PWM, PD-PWM, and PODHPWM are described. Then, PD-HPWM is proposed via a carrier based realization. By means of the simulations of a $6 \mathrm{MW}$ wind turbine medium voltage grid-side converter as $3 \mathrm{~L}-$ NPC-VSC and 3L-HB-VSC, the equivalency between these POD methods and the equivalency between these PD methods are demonstrated via output voltage/current waveforms. Also, the switch current waveforms illustrates the similarity of the switch utilizations in the two VSCs. Next, experimental results of a $1.5 \mathrm{~kW}$ prototype validate the performance of the proposed PD-HPWM. Finally, the advantages and disadvantages of 3L-HB-VSC with PD-HPWM compared to 3L-NPC-VSC are discussed and its possible application fields are addressed.

\section{THREE-LEVEL PWM METHODS}

For 3L-NPC-VSCs, there are two types of triangular carrier based PWM methods: POD-PWM and PD-PWM (Fig. 3 and 4 for phase- $a$ ). Both methods compare the reference voltage $v^{*}{ }_{a}$ with the upper triangle $v_{\text {tri }}$ and the lower triangle $v_{\text {tri- }}$ signals where $v_{\text {tri }}$ and $v_{\text {tri- }}$ 's absolute peak values are scaled with the half of the total DC bus voltage of the converter, $V_{D C}$. Illustrated in Fig. 5, the difference between the two methods is that $v_{t r i+}$ and $v_{\text {tri- }}$ for PD-PWM are in-phase while they have $180^{\circ}$ phase shift for POD-PWM [11]. These two methods results in similar output voltage and current in single-phase applications; however, the elimination of common mode harmonic (zero sequence) components in the line-to-line output voltage in three-phase systems makes the output difference favoring PD-PWM [11].

In 3L-HB-VSCs, the single leg switched or hybrid PWM method (Fig. 6) modulates the leg of $S A 1 \& S A 2$ with the switching frequency $f_{S}$ while the other leg of $S A 3 \& S A 4$ with the electrical frequency $f_{e}[11],[13]$. Hence, the switches are utilized similarly to those of the NPC for $\mathrm{PF} \approx 1$. Also, the resulting output current waveform is theoretically identical to the one with POD-PWM because the comparison of the absolute value of the reference voltage $\left|v_{a}^{*}\right|$ with the triangular carrier signal $v_{t r i}$ works similarly to POD-PWM. Thus, this method will be called POD-HPWM hereafter in this paper. Nonetheless, the existence of the PD counterpart of PODHPWM would carry more importance due to its favorable output performance in three-phase applications. Hence, PDHPWM (Fig. 7) is proposed as follows. The modulation algorithm compares the positive part of $v_{a}^{*}$ with $v_{\text {tri+ }}$ for $v_{a}^{*}>0$ and the negative part of $v_{a}^{*}$ with $v_{t r i-}$ for $v_{a}^{*}<0$ in order to modulate the leg with the switches SA1\&SA2 of the HB while the polarity of $v_{a}{ }_{a}$ is used for the switching decision of the leg with $S A 3 \& S A 4$. The signals employed in the hybrid PWM methods are illustrated in Fig. 8.

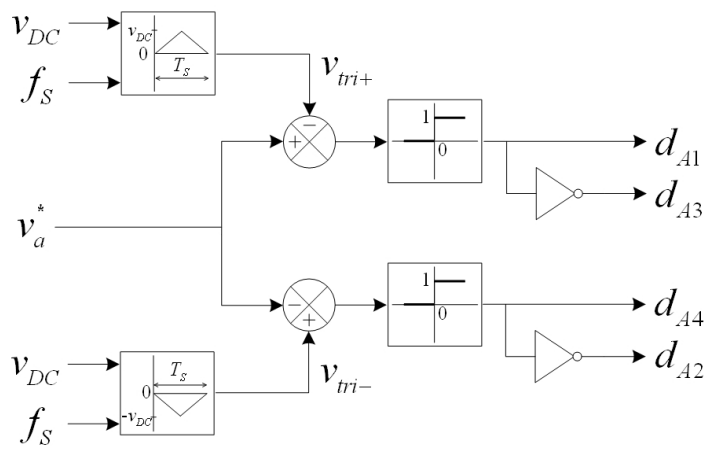

Fig. 3. POD-PWM algorithm for phase- $a$ of $3 \mathrm{~L}-\mathrm{NPC}-\mathrm{VSC}$

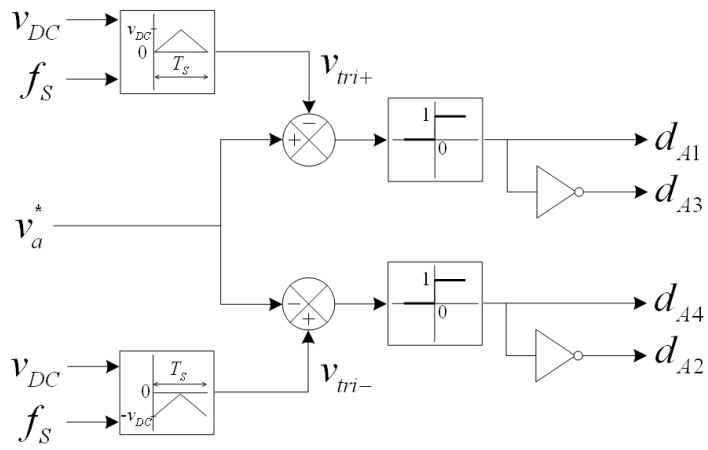

Fig. 4. PD-PWM algorithm for phase- $a$ of 3L-NPC-VSC

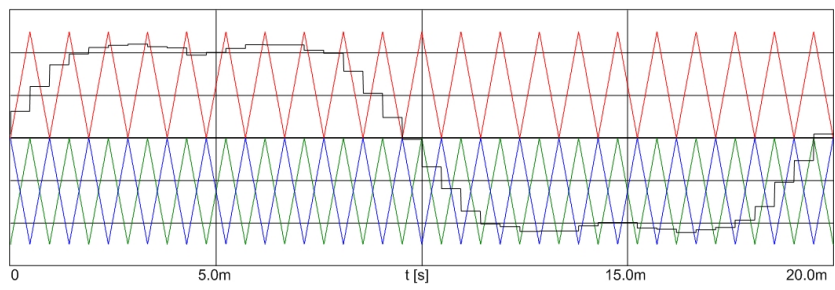

Fig. 5. The waveforms of POD-PWM and PD-PWM employing thirdharmonic added reference voltage $v_{a}^{*}$ (black), $v_{t r i+}$ (red), $v_{t r i-}$ for PD-PWM (green), and $v_{t r i-}$ for PD-PWM (blue) 


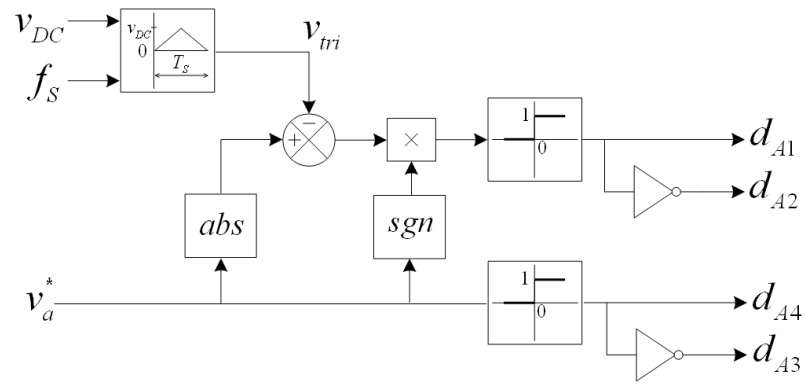

Fig. 6. POD-HPWM algorithm for phase- $a$ of 3L-HB-VSC

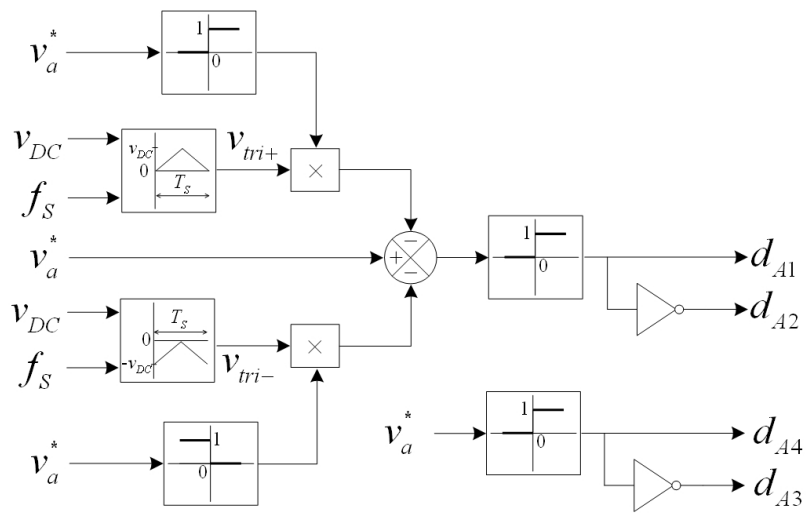

Fig. 7. PD-HPWM algorithm for phase- $a$ of 3L-HB-VSC

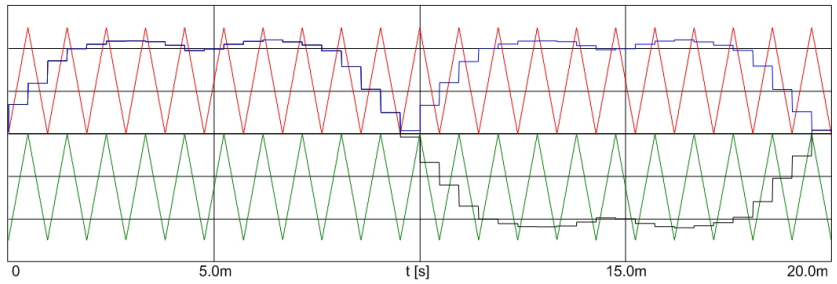

Fig. 8. The waveforms of POD-HPWM and PD-HPWM employing thirdharmonic added reference voltage $v_{a}^{*}$ (black), $\left|v_{a}^{*}\right|$ (blue), $v_{t r i}$ and $v_{t r i+}$ (red), and $v_{\text {tri- }}$ (green).

\section{SiMULATION RESULTS}

The simulations are conducted by employing ideal component models built in Ansoft/Simplorer for the medium voltage 3L-HB-VSC and 3L-NPC-VSC shown in Fig. 1 and 2 for a grid connection of $6 \mathrm{MW}$ wind turbine via a three-phase transformer with open primary windings. The simulation parameters are given in Table I. In implementation of PWM algorithms, asymmetrical double-edge regular sampling is used [11]. Fig. 9 demonstrates that the output current is significantly less distorted for PD-PWM than POD-PWM in 3L-NPC-VSC. The line-to-line voltage waveforms shown in Fig. 10 explain the performance difference such that PD-PWM results only in the voltage steps of $V_{D C}$ while POD-PWM results in both $V_{D C}$ and $2 V_{D C}$. For 3L-HB-VSC, Fig. 11 demonstrates the equivalencies between POD-PWM and POD-HPWM and between PD-PWM and PD-HPWM. Table II summarizes the output current Total Harmonic Distortion $\mathrm{THD}_{\mathrm{I}}$ values for each case. Demonstrated in Fig. 12, there is a correlation between the switch utilizations of 3L-NPC-VSC and 3L-HB-VSC with the hybrid PWM methods. Given in Table III, for $\mathrm{PF} \approx 1$, there is one-to-oneness for IGBT utilization while the antiparallel diodes $D A 2 \& D A 1$ of the $\mathrm{HB}$ converter are utilized similarly to the clamping diodes $D A 5 \& D A 6$, respectively.

TABLE I

SIMULATION PARAMETERS

\begin{tabular}{|l|l|}
\hline Grid line-to-line voltage & $10 \mathrm{kV}_{\mathrm{rms}}(50 \mathrm{~Hz})$ \\
\hline Filter inductor, $L$ & $450 \mu \mathrm{H}(10 \%)$ \\
\hline Transformer turns-ratio, $N\left(N_{\text {inv }} / N_{\text {grid }}\right)$ & $0.3(1: 3.33)$ \\
\hline Output power, $P$ & $6 \mathrm{MW}$ \\
\hline Power factor, PF & 1 \\
\hline DC bus voltage, $V_{D C}$ & $2500 \mathrm{~V}$ \\
\hline Switching frequency, $f_{S}$ & $1050 \mathrm{~Hz}$ \\
\hline
\end{tabular}

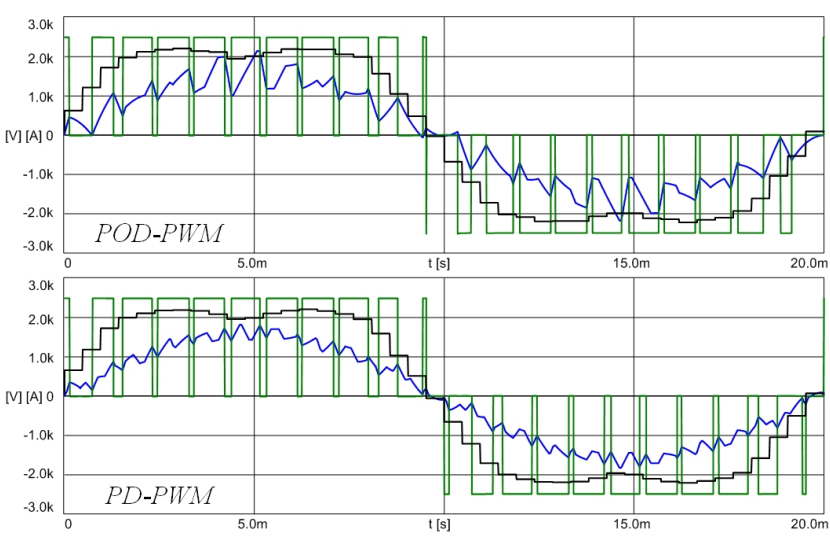

Fig. 9. The reference voltage $v_{a}{ }_{a}$ (black), output phase voltage $v_{a}$ (green), and output current $i_{a}$ (blue) waveforms of phase- $a$ for POD-PWM (upper) and PD-PWM (lower) in 3L-NPC-VSC

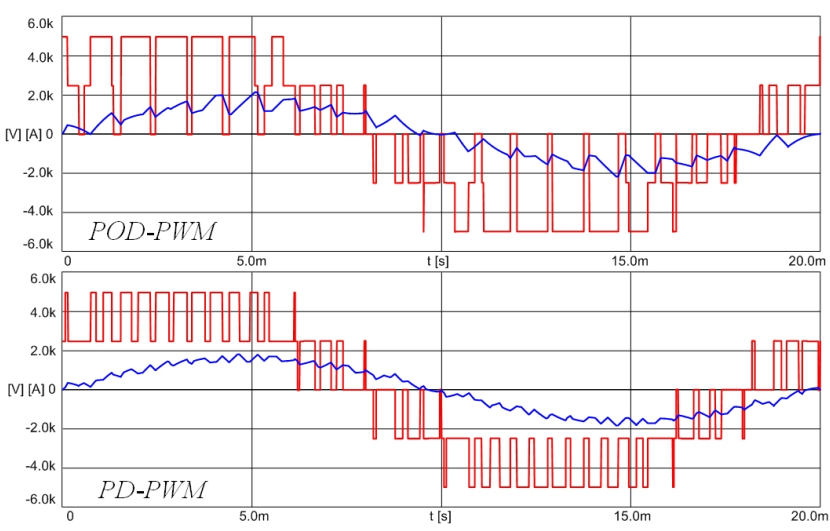

Fig. 10. The output line-to-line voltage $v_{a b}$ (red) and output current $i_{a}$ (blue) waveforms for POD-PWM (upper) and PD-PWM (lower) in 3L-NPC-VSC

TABLE II

THD $_{\text {I RESULTS IN SIMULATIONS }}$

\begin{tabular}{|l|c|c|c|c|}
\hline VSC & \multicolumn{2}{|c|}{ NPC } & \multicolumn{2}{c|}{ HB } \\
\hline Modulation & $\begin{array}{c}\text { POD- } \\
\text { PWM }\end{array}$ & $\begin{array}{c}\text { PD- } \\
\text { PWM }\end{array}$ & $\begin{array}{c}\text { POD- } \\
\text { HPWM }\end{array}$ & $\begin{array}{c}\text { PD- } \\
\text { HPWM }\end{array}$ \\
\hline THD $_{\mathrm{I}}(\%)$ & 19.63 & 8.88 & 19.58 & 8.78 \\
\hline
\end{tabular}




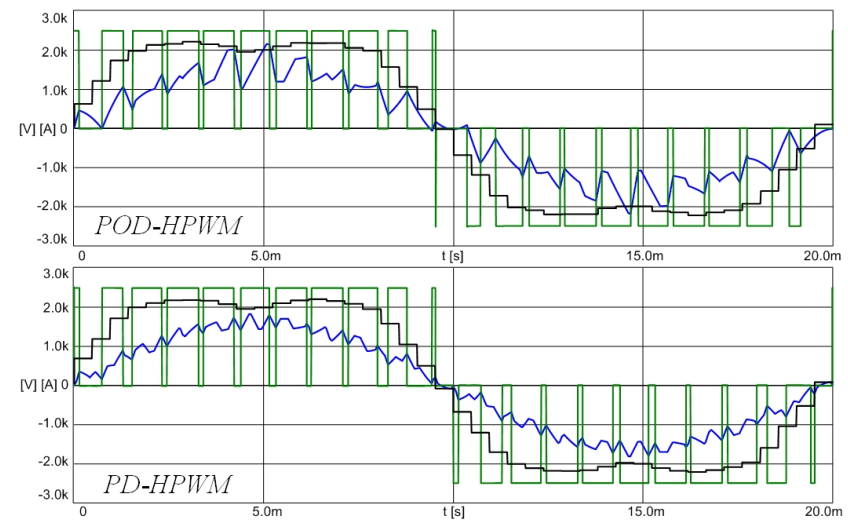

Fig. 11. The reference voltage $v_{a}{ }_{a}$ (black), output phase voltage $v_{a}$ (green), and output current $i_{a}$ (blue) waveforms of phase- $a$ for POD-HPWM (upper) and PD-HPWM (lower) in 3L-HB-VSC

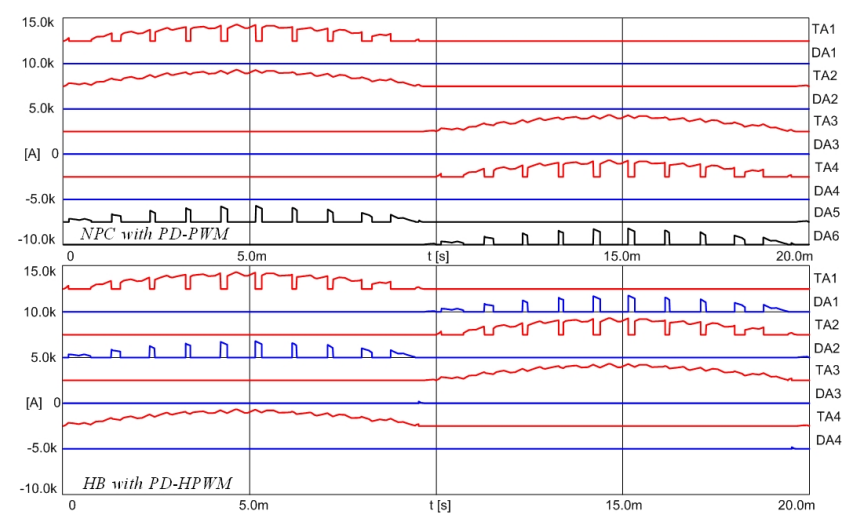

Fig. 12. IGBT (red), antiparallel diode (blue), and neutral point clamping diode (black) current waveforms with 2500A offsets for phase- $a$ of 3L-NPC-

VSC with PD-PWM (upper) and 3L-HB-VSC with PD-HPWM (lower)

TABLE III

CORRELATION OF SWITCH UTILIZATION IN 3L-HB- AND -NPC-VSCS

\begin{tabular}{|c|c|c|c|c|c|c|}
\hline NPC & TA1 & TA2 & TA3 & TA4 & & \\
\hline HB & TA1 & TA4 & TA3 & TA2 & & \\
\hline NPC & DA1 & DA2 & DA3 & DA4 & DA5 & DA6 \\
\hline HB & DA4 & DA4 & DA3 & DA3 & DA2 & DA1 \\
\hline
\end{tabular}

\section{EXPERIMENTAL RESULTS}

The experiments are conducted via a prototype circuit with the parameters listed in Table IV. The prototype HB and NPC circuits differ from the circuits given in Fig. 1 and 2 by the usage of a three-phase RC load instead of an electricity grid. Illustrated by the oscilloscope waveforms in Fig. 13 and 14, PD-PWM results in the output current with significantly less harmonic distortion than POD-PWM in 3L-NPC-VSC. Due to the finite amount of transformer leakage inductance, these current waveforms are slightly less distorted than the simulation waveforms. Fig. 15 and Fig. 16 show that 3L-HBVSC with POD-HPWM and the proposed PD-HPWM can generate output waveforms very close to those obtained by $3 \mathrm{~L}-$ NPC-VSC. The deviation on the output current waveform during the zero crossings is caused by a synchronization problem related with loading and processing duty cycles in the DSP control platform and can be avoided by DSP code improvements. Fig. 17 shows that one leg of the HB is continuously switched while the other leg is switched twice in an electrical period.

TABLE IV

EXPERIMENTAL SETUP PARAMETERS

\begin{tabular}{|l|l|}
\hline Load line-to-line voltage & $220 \mathrm{~V}_{\mathrm{rms}}(50 \mathrm{~Hz})$ \\
\hline Filter inductor, $L$ & $11 \mathrm{mH}(10 \%)$ \\
\hline Transformer turns-ratio, $N\left(N_{\text {inv }} / N_{\text {grid }}\right)$ & 1 \\
\hline Load capacitance, $C$ & $5.6 \mu \mathrm{F}$ \\
\hline Load resistance, $R$ & $33 \Omega$ \\
\hline Output power, $P$ & $1.5 \mathrm{~kW}$ \\
\hline Power factor, PF & $\sim 1$ \\
\hline DC bus voltage, $V_{D C}$ & $180 \mathrm{~V}$ \\
\hline Switching frequency, $f_{S}$ & $1050 \mathrm{~Hz}$ \\
\hline
\end{tabular}

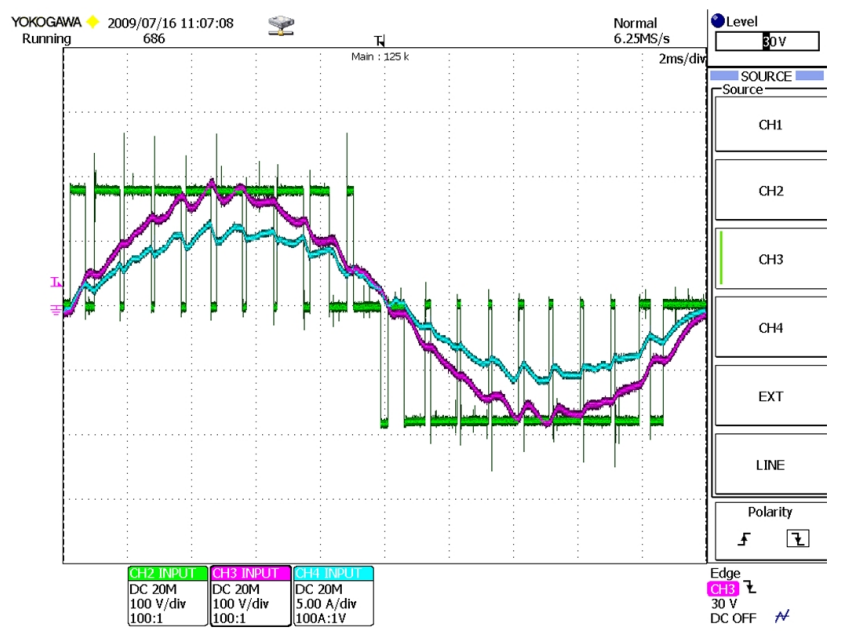

Fig. 13. The load phase voltage (red), output phase voltage (green), and output current (blue) waveforms of phase- $a$ for POD-PWM in 3L-NPC-VSC

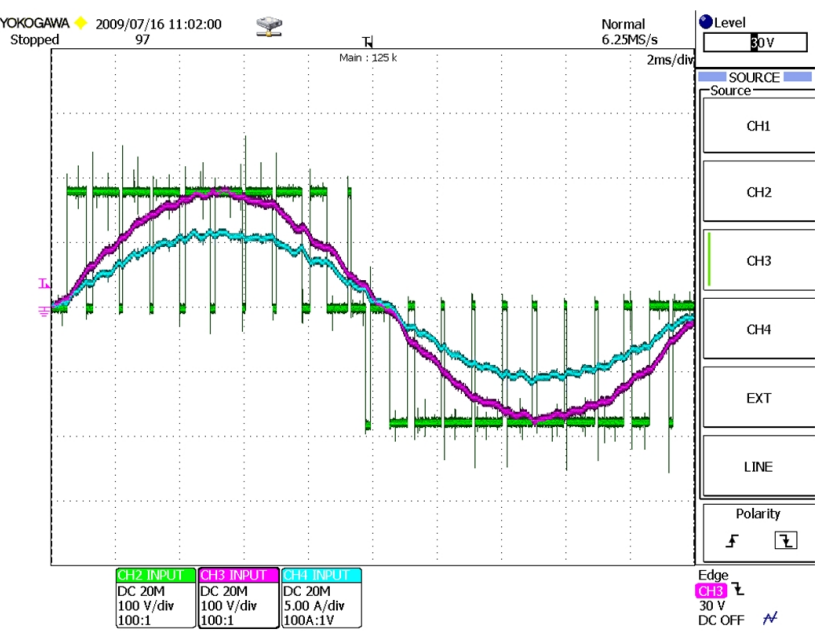

Fig. 14. The load phase voltage (red), output phase voltage (green), and output current (blue) waveforms of phase- $a$ for PD-PWM in 3L-NPC-VSC 


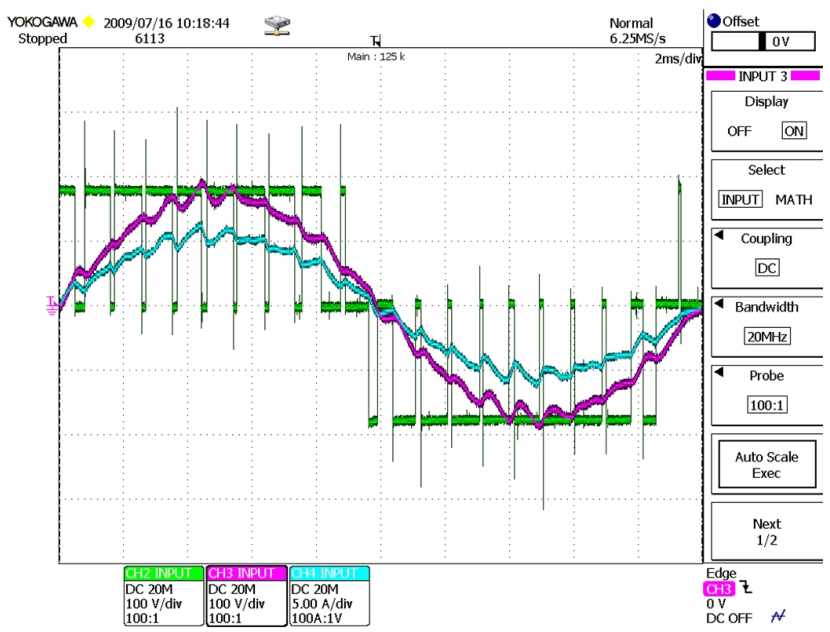

Fig. 15. The load phase voltage (red), output phase voltage (green), and output current (blue) waveforms of phase- $a$ for POD-HPWM in 3L-HB-VSC

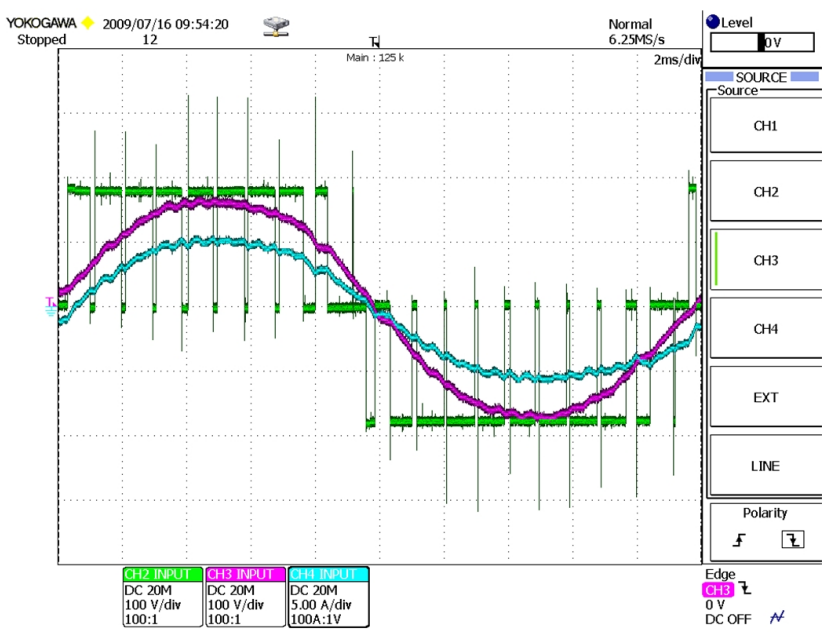

Fig. 16. The load phase voltage (red), output phase voltage (green), and output current (blue) waveforms of phase- $a$ for PD-HPWM in 3L-HB-VSC

\section{DISCUSSION}

The proposed PD-HPWM carries importance due to its equivalent performance and similar switch utilization to $3 \mathrm{~L}$ NPC-VSC, which is dominantly used converter topology especially for medium voltage converters with low switching frequency $\left(f_{S}<2 \mathrm{kHz}\right)$. Hence, for medium voltage grid converter applications requiring transformers or for open winding machine converter applications, 3L-HB-VSC can be considered as an alternative to 3L-NPC-VSC because the former has simpler, more modular, and more reliable $2 \mathrm{~L}$ structure than the $3 \mathrm{~L}$ structure. Also, the clamping diodes and neutral point variation problem of the NPC are eliminated in the HB. Considering switch utilization, the duty of the clamping diodes in the NPC is performed by the antiparallel diodes in the HB. However, for the applications where IGBTs and diodes are packaged together, the elimination of the clamping diodes increases the power loss stress on the IGBTdiode packages forming the switched HB legs and becomes a disadvantage. On the other hand, for medium voltage applications where these two semiconductors are packaged separately, this elimination is an advantage of reducing semiconductor cost and more efficient utilization of the switches. Moreover, several control and design strategies can be developed by means of hybrid modulation of 3L-HB-VSCs as follows.

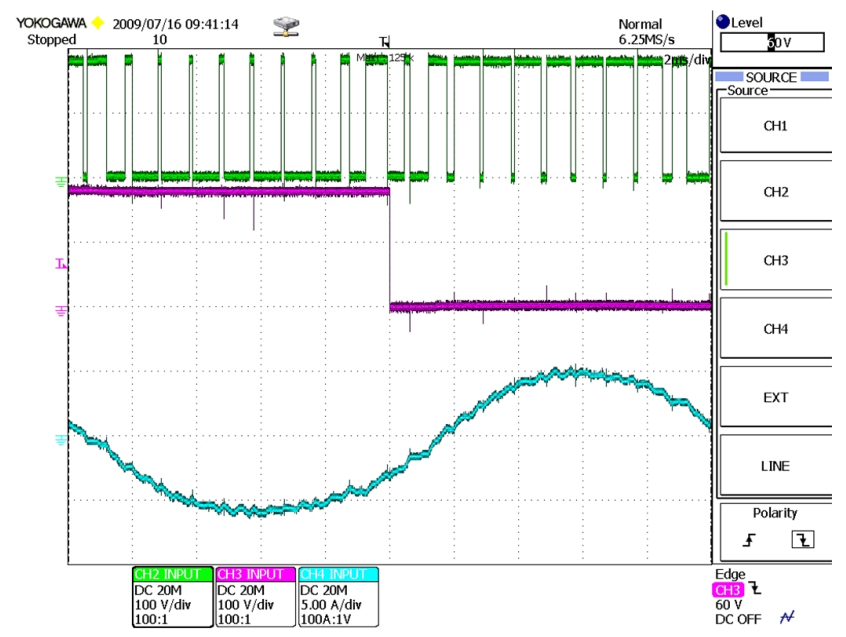

Fig. 17. With respect to the negative rail of the DC bus, the switched leg's voltage (green) and the other leg's voltage (red), and output current (blue) waveforms of phase- $a$ for PD-HPWM in 3L-HB-VSC

The switched leg and the other leg can be interchanged cyclically. Hence, better utilization of the switches over the NPC can be achieved and better lifetime for each switch can be attained. Also, as proposed in [9], different type of switches can be used for the two legs. For example, the switches with low switching loss for the switched leg and the switches with low conduction loss for the other leg can be chosen in order to optimize the efficiency.

For the grid applications requiring capacitive switching ripple filters, the implementation of these filters at the grid side of the transformer does not influence the output performance equivalency of the two converters. However, if a capacitive filter is directly applied to the inverter side of a $3 \mathrm{~L}-\mathrm{HB}-\mathrm{VSC}$, then the elimination of the common mode terms in three-phase system does no longer exist and the converter output current deteriorates significantly with the harmonics around the switching frequency. In order to solve this problem, common mode inductors in series with the filter inductors can be applied to 3L-HB-VSC.

\section{CONCLUSION}

The 3L-HB-VSC with the proposed PD-HPWM produces the same output performance as the 3L-NPC-VSC with PDPWM or space vector NTV. Moreover, the PD-HPWM enables the former VSC to utilize its switches similarly to those of the latter. The performance of the proposed method has been validated by the simulations and experiments. Thus, the former VSC can be considered as an alternative to the latter for medium voltage grid-connected converter with transformer 
or open winding motor drive applications in regards to design simplicity and switch utilization flexibility. Only disadvantage, which can be avoided by the hardware solutions discussed, is the limitation of capacitive switching ripple filter employment for grid-connected applications.

\section{ACKNOWLEDGMENT}

This work was supported by Aalborg University-Vestas Wind Systems partnership under Vestas Power Program. Any opinions, findings, and conclusions or recommendations expressed in this material are those of the authors and do not necessarily reflect those of Vestas Wind Systems.

The authors gratefully acknowledge the contributions of $\mathrm{M}$. Swierczynski, A. Adamczyk, and T. Kerekes for their work on the experimental test setup.

\section{REFERENCES}

[1] N. Mohan, T. M. Undeland, W. P. Robbins, Power Electronics Converters, Applications, and Design, $4^{\text {th }}$ Edition, NJ: John Wiley \& Sons, 2003.

[2] S. B. Kjaer, J. K. Pedersen, F. Blaabjerg, "A review of single-phase gridconnected inverters for photovoltaic modules," IEEE Trans. on Ind. Applicat., Vol. 41, No. 5, pp.1292-1306, Sept./Oct. 2005.

[3] F. Z. Peng, H. Akagi, A. Nabae, "A new approach to harmonic compensation in power systems - a combined system of shunt passive and series active filters," IEEE Trans. on Ind. Applicat., Vol. 26, No. 6, pp. 983-990, Nov.r/Dec. 1990

[4] H. Kim, S. K. Sul, "Compensation voltage control in dynamic voltage restorers by use of feed forward and state feedback scheme," IEEE Trans. on Power Electron., Vol. 20, No. 5, pp. 1169-1177, Sept. 2005.

[5] S. Ponnaluri, J. K. Steinke, P. Steimer, S. Reichert, B. Buchmann, "Design comparison and control of medium voltage STATCOM with novel twin convertert topology," in Proc. 2004 IEEE PESC.

[6] K. Gopakumar, V. T. Ranganathan, S. R. Bhat, "Split-phase induction motor operation from PWM voltage source inverter," IEEE Trans. on Ind. Applicat., Vol. 29, No. 5, pp. 927-932, Sept./Oct. 1993.

[7] M. R. Baiju, K. K. Mohapatra, R. S. Kanchan, K. Gopakumar, "A dual two-level inverter scheme with common mode voltage elimination for an induction motor drive," IEEE Trans. on Power Electron., Vol. 19, No. 3, pp. 794-805, May 2004.

[8] D. Casadei, G. Grandi, A. Lega, C. Rossi, "Multilevel operation and input power balancing for a dual two-level inverter with insulated DC sources," IEEE Trans. on Ind. Applicat., Vol. 44, No. 6, pp. 1815-1824, Nov./Dec. 2008.

[9] K. A. Corzine, S. D. Sudhoff, C. A. Whitcomb, "Performance characteristics of a cascaded two-level converter," IEEE Trans. on Energy Conversion, Vol. 14, No. 3, pp. 433-439, Sept. 1999.

[10] Y. Zhao, T. A. Lipo, "Space vector PWM control of dual three-phase induction machine using vector space decomposition," IEEE Trans. on Ind. Applicat., Vol. 31, No. 5, pp. 1100-1109, Sept./Oct. 1995.

[11] D. G. Holmes, T. A. Lipo, Pulse Width Modulation for Power Converters, Piscataway, NJ: IEEE-Wiley, 2003.

[12] B. Wu, High-Power Converters and AC Drives, Piscataway, NJ: IEEE Press, 2006

[13] R. S. Lai and K. D. T. Ngo, "A PWM method for reduction of switching loss in a full-bridge inverter," IEEE Trans. on Power Electron., Vol. 10, No. 3, pp. 326-332, May 1995.

[14] A. Nabae, I. Takahashi, H. Akagi, "A new neutral-point-clamped PWM inverter," IEEE Trans. on Ind. Applicat., Vol. IA-17, No.5, pp. 518-523, Sept./Oct. 1981. 